\title{
Diagnosis of Streptococcus pneumoniae pneumonia by quantitative enzyme linked immunosorbent assay of C-polysaccharide antigen
}

\author{
S H Gillespie, M D Smith, A Dickens, J G Raynes, K P W J McAdam
}

\begin{abstract}
Aims-To evaluate the use of a quantitative enzyme linked immunosorbent assay (ELISA) detecting C-polysaccharide (PnC) antigen in sputum for the diagnosis of Streptococcus pneumoniae infection.

Methods-Specimens of sputum from 60 patients with acute community and hospital acquired pneumonia and infective exacerbations of obstructive airways disease were examined by semiquantitative culture and antigen ELISA.

Results-Using a cutoff value of $1 \mu \mathrm{g} / \mathrm{ml}$ PnC antigen for a positive result, the sensitivity of this assay was $90 \cdot 3 \%$, specificity $93.1 \%$, predictive value of a positive result was $93 \cdot 5 \%$, and the predictive value of a negative result $89 \cdot 6 \%$.

Conclusions-Quantitation of C-polysaccharide antigen in sputum by ELISA distinguishes between carriage of oral bacteria which express PnC-like antigen and infection with $S$ pneumoniae and compares favourably with other diagnostic methods.
\end{abstract}

(F Clin Pathol 1994;47:749-751)

The diagnosis of pneumococcal infection has been attempted using methods which detect the species specific C-polysaccharide (PnC) antigen by latex agglutination, coagglutination, and enzyme linked immunosorbent assay (ELISA).$^{1-5}$ Doubts about the efficacy of this technique are raised on the grounds that many other organisms share the immunodominant epitope-phosphorylcholine. These organisms include protozoa ${ }^{6}$ (the excreted factor of Leishmania donovani), helminths (Toxocara canis excretory secretory antigen, Ascaris suum) ${ }^{78}$ and fungi (Aspergillus spp and Mentagrophytes spp)..$^{9}$ Of more importance is cross-reaction with other $a$ haemolytic streptococci ${ }^{10-12}$ and group $C \beta$ haemolytic strepto$\operatorname{cocci}^{10}$ as these organisms form part of the normal oral flora and this could lead to false positive results in sputum samples.

Studies of cross-reaction in $a$ haemolytic streptococci have shown that up to $36 \%$ bind anti-PnC antibodies. ${ }^{12}$ Cross-reaction is more common in clinical isolates rather than stock cultures, although the speciation of positive isolates in this study was incomplete. Lower concentrations of antigen were found in the $a$ haemolytic streptococci, suggesting that diagnosis of pneumococcal infection by $\mathrm{PnC}$ detection may be valuable in the clinical setting. ${ }^{12}$ We have recently shown that PnC-like antigen is found in the $S$ oralis group of $a$ haemolytic streptococci (comprising $S$ oralis, $S$ mitis, and $S$ pneumoniae) and that this antigen is released more readily from $S$ pneumoniae and is present at higher concentrations in the latter organism. ${ }^{13}$ This suggests that by quantification of $\mathrm{PnC}$ antigen in sputum it might be possible to distinguish between cross-reacting antigens found in oral streptococci from infection with $S$ pneumoniae. Diagnosis by quantifying $\mathrm{PnC}$ antigen has been shown to be valuable in a small study of 12 patients with $S$ pneumoniae pneumonia. ${ }^{14}$ We have, therefore, sought to evaluate a sensitive quantitative $\mathrm{PnC}$ antigen detection ELISA in a clinical setting using a larger group of patients.

\section{Methods}

Patients with the following diagnoses were selected: pneumonia with and without bacteraemia, hospital acquired pneumonia, and infective exacerbations of obstructive airways disease. Specimens were collected from 60 patients admitted to four hospitals in North London with suspected acute lower respiratory tract infection. The laboratory request forms and clinical case records were consulted to obtain details of the patients' clinical presentation.

Specimens of sputum were collected, cultured immediately, and then stored at $-70^{\circ} \mathrm{C}$ until tested in the PnC antigen ELISA. A Gram stain was performed on a purulent portion of the sputum specimen. The sputum was examined for quality by light microscopy under low power ( $\times 10$ objective). Salivary contamination was detected by noting the presence of buccal squamous cells on a scale of $0-3$. These figures represented, respectively, 0 no squamous cells seen, 1 few squamous cells seen, but many clear fields, 2 squamous cells found in small numbers in many fields, and 3 squamous cells found in large numbers in most fields. Similarly, purulence was determined by noting the presence of pus cells on a scale of $0-3$. These figures represented 0 no pus cells seen, 1 pus cells seen on only a few fields, 2 pus cells seen in most fields in moderate numbers, and 3 pus cells present in most fields in large numbers. Specimens with a purulence score of one or less and a contamination score of one or more were not included in this study.

Sputum was diluted 1 in 5 in $2.5 \% \mathrm{w} / \mathrm{v}$ 
Concentration of $\mathrm{PnC}$ antigen in the sputum of 60 patients with acute lower respiratory tract infection.

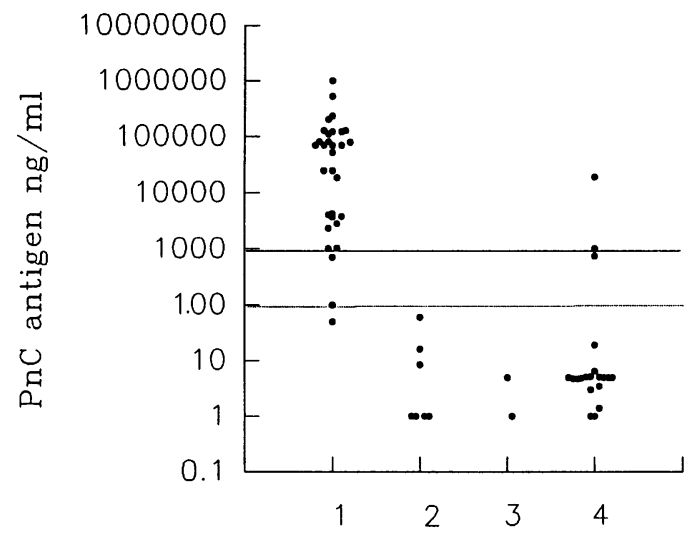

$\mathrm{N}$-acetyl-cysteine and homogenised for 15 minutes at room temperature. An aliquot of $100 \mu \mathrm{l}$ was taken and diluted in $9.9 \mathrm{ml}$ Ringer's solution, and a second dilution made $(100 \mu \mathrm{l}$ in $9.9 \mathrm{ml})$. An aliquot of $100 \mu \mathrm{l}$ from each dilution was inoculated on to blood agar and a second aliquot on to chocolated blood agar. Both plates were incubated at $37^{\circ} \mathrm{C}$ in an atmosphere of $5-10 \% \mathrm{CO}_{2}$ and increased humidity. The number of colony forming units for each morphological type was recorded. Identification of isolates to species level was achieved using conventional methods. An isolate was considered "significant" if there were more than 20 colony forming units on the second dilution plate (equivalent to approximately $10^{7} \mathrm{cfu} / \mathrm{ml}$ in the original specimen).

The quantitative $\mathrm{PnC}$ antigen capture ELISA has been described before. ${ }^{13}$ Briefly, 96-well microtitration plates (M29A Dynatech, UK) were coated with a 1 in 2000 dilution of a mouse IgM monoclonal antiphosphorylcholine antibody (five of 88) (Universal Biologicals, UK) in $0.06 \mathrm{M}$ bicarbonate buffer ( $\mathrm{pH} 9.6)$ by overnight incubation at $4^{\circ} \mathrm{C}$. After washing with $10 \mathrm{mM}$ TRIS containing $0.15 \mathrm{M} \mathrm{NaCl}$ and Tween-20 $(0.05 \%)$ and $1 \mathrm{mM} \mathrm{CaCl}$ (TBSTC) samples were added and incubated for one hour at room temperature. After four further washes $100 \mu \mathrm{l} \mathrm{C}$-reactive protein $(1.7 \mu \mathrm{g} / \mathrm{ml})$ conjugated to horseradish peroxidase diluted in TBSTC was added. The plates were incubated for three hours at room temperature and then washed. 2,2'-azino-di-3-ethylbenzthiazoline sulphonate ABTS peroxidase substrate (Kirkegaard-Perry, Gaithersburg, Maryland, USA) was added and the optical density read at $405 \mathrm{~nm}$ using an automated ELISA reader (Titertek Multiscan MC, Flow

Evaluation of quantitative PnC ELISA, differences in sensitivity, specificity, predictive value of a positive test, and predictive value of a negative test with differing cut-off concentrations of $P_{n} C$ antigen

\begin{tabular}{llll}
\hline & \multicolumn{3}{l}{ Per cent for cutoff values } \\
\cline { 2 - 4 } Measure & $100(\mathrm{ng} / \mathrm{ml})$ & $500(\mathrm{ng} / \mathrm{ml})$ & $1(\mu \mathrm{g} / \mathrm{ml})$ \\
\hline Sensitivity & $96 \cdot 7$ & $93 \cdot 3$ & $90 \cdot 3$ \\
Specificity & $89 \cdot 6$ & $89 \cdot 6$ & $93 \cdot 1$ \\
Predictive value of positive & $90 \cdot 9$ & $93 \cdot 5$ & $93 \cdot 5$ \\
Predictive value of negative & $96 \cdot 2$ & $92 \cdot 8$ & $89 \cdot 6$ \\
\hline
\end{tabular}

Laboratories). Samples of sputum processed as above were diluted in TBSTC in four 10fold dilutions and each dilution tested in duplicate. The concentration of PnC antigen was determined by comparison with a standard curve of known concentrations of purified $\mathrm{PnC}$ antigen tested on the same plate.

\section{Results}

Of the 60 patients included, 26 had a previous history of chronic obstructive airways disease during an acute exacerbation, 24 had acute community acquired pneumonia, and 10 were patients with acute hospital acquired pneumonia. There were 45 men and 15 women The mean age was 64.4 (range $22-88$, median 64). A history of previous antibiotic treatment was obtained in 12 cases. In 10 cases the prescription of antibiotics before admission could not be determined.

All specimens were purulent with a mean purulence score of 2.33 and a mean epithelial cell score of 0.72 . Of the 60 patients, 31 had a clinically important isolate of $S$ pneumoniae. Of these, six had a mixed infection with Haemophilus influenzae. Seven patients had a clinically important isolate of $H$ influenzae alone and two of Moraxella catarrhalis alone. In the remaining 20 patients no unequivocal bacterial pathogens were isolated.

The patients were divided into four groups on the basis of their bacteriological diagnosis. These were $S$ pneumoniae, $H$ influenzae, $M$ catarrhalis and no relevant isolate. The mean concentration of $\mathrm{PnC}$ antigen detected in each group was as follows: $S$ pneumoniae $103.4 \mu \mathrm{g} / \mathrm{ml}$ (range $0.05-1000 \mu \mathrm{g} / \mathrm{ml}$, median $70 \mu \mathrm{g} / \mathrm{ml}$ ), $H$ influenzae $0.012 \mu \mathrm{g} / \mathrm{ml}$ (range 0.001-0.06 $\mu \mathrm{g} / \mathrm{ml}$, median $0.03 \mu \mathrm{g} / \mathrm{ml}), M$ catarrhalis $0.005 \mu \mathrm{g} / \mathrm{ml}$, and for those patients with no clinically important bacterial isolate $1.06 \mu \mathrm{g} / \mathrm{ml}$ (range $0.001-19 \mu \mathrm{g} / \mathrm{ml}$, median $0.05 \mu \mathrm{g} / \mathrm{ml}$ ). These data are illustrated in the figure showing individual points for each specimen and lines showing two potential cutoff values.

To define whether there is a difference between the result of quantitative PnC ELISA from the patients infected with $S$ pneumoniae and those who were not infected with this organism, all of the other bacterial diagnostic groups were combined, a logarithmic transformation was performed, and an unpaired $t$ test applied. The results of this show that the difference between the means is $(\log ) 2.404$ (95\% confidence interval 2.89 to 1.91 ), a difference which is significant at $\mathrm{p}<0.00001$. The Mann-Whitney U test for non-parametric data was also applied to the $S$ pneumoniae group and to the combined non-S pneumoniae group. The difference between the medians was $69995, \kappa=318$, with $95 \%$ confidence interval 4199-79995. The $U$ test was $19 \cdot 5$, the $\mathrm{z}$ statistic was -6.38 with a two-tailed probability of $<0.00001$.

The sensitivity, specificity, and predictive value of a positive and negative test were calculated using three different cutoff values. These results are found in the table. 


\section{Discussion}

The diagnosis of $S$ pneumoniae by non-quantitative PnC detection methods has been reported before. ${ }^{15}$ In a prospective study comparing three non-quantitative diagnostic methods in 249 patients with community acquired pneumonia, 63 patients had positive bacterial culture with $S$ pneumoniae. The sensitivity of the test was as follows: PnC-ELISA 49\%, PnC-coagglutination (PnC-CoA) 65\%; and capsular polysaccharide coagglutination (CPS-CoA) $63 \%$. The specificity was $98 \%$ for all three methods. PnC antigen was found almost as often in non-purulent samples as purulent samples and in both washed and unwashed samples. The sensitivity of both CoA methods was lowered by antimicrobial treatment before admission. ${ }^{16}$ Diagnosis by detection of PnC antigen in saliva of patients with acute community acquired pneumonia has also been reported. ${ }^{2}$ In this study there was clinical and radiological evidence of pneumonia in 65 patients and $S$ pneumoniae was isolated in 29 cases. PnC antigen was found in the saliva of 16 of these patients (sensitivity of $55 \%$, with only one false positive reaction). ${ }^{2}$ The low sensitivity reported in these studies together with a high specificity suggests that a positive result was only reported when substantial quantities of $\mathrm{PnC}$ antigen were detected.

The lower limit of detection of $\mathrm{PnC}$ with the ELISA reported here is $1 \mathrm{ng} / \mathrm{ml}$, a level of antigen which can be found in many patients as a result of cross-reacting antigens from oral bacteria. It would be possible, therefore, to define almost all of the patients "positive" for $\mathrm{PnC}$ antigen. By varying the cutoff value it was possible to set the level which optimised the sensitivity and specificity of the test. Using a cutoff value of $1 \mu \mathrm{g} / \mathrm{ml}$, it was possible to obtain a sensitivity of $90 \cdot 3 \%$, a specificity of $93 \cdot 1 \%$, a predictive value of a positive result of $93.5 \%$ and a predictive value of a negative result of $89.6 \%$ when compared with the result of semiquantitative bacteriological culture. This implies that quantitation is successful in minimising false positive results arising from PnC-like antigens possessed by oral streptococci and bacteria contaminating saliva.

The sensitivity of quantitative PnC ELISA compares favourably with other methods for the diagnosis of $S$ pneumoniae pneumonia. This includes methods which depend on detection of capsular polysaccharide antigen or C-polysaccharide antigen. ${ }^{1-416}$

Another problem, however, with assays detecting PnC antigen is cross-reaction with some strains of $H$ influenzae (manuscript in preparation). No false positive results were obtained in this study in the seven patients infected with $H$ influenzae alone. Previous reports have noted false positive reactions in $1-2 \%$ of patients infected with $H$ influenzae. ${ }^{34}$ We conclude that quantitative antigen detection ELISA for PnC can be used to distinguish infection from colonisation with oral streptococci expressing C-polysaccharide-like antigens. The principle of quantitative antigen detection for the diagnosis of $S$ pneumoniae in sputum might also be applied to other pneumococcal antigens where cross-reactions are less common.

We gratefully acknowledge the technical assistance of Miss Shila Patel.

1 Holmberg H, Krook A. Comparison of enzyme-linked immunosorbent assay with coagglutination and latex agglutination for rapid diagnosis of pneumococcal pneumonia by detecting antigen in sputa. Eur 7 Clin Microbiol 1986;5:282-6.

2 Krook A, Fredlund H, Holmberg H. Diagnosis of pneumococcal pneumonia by detection of antigen in saliva. Eur f Clin Microbiol 1986;5:639-42.

3 Krook A, Holmberg H, Sjogren AM. A new coagglutination test for detecting pneumococcal C-polysaccharide. Eur f Clin Microbiol 1987;6:68-9.

4 Holmberg H, Holme T, Krook A, Olsson T, Sjoberg L, Sjogren AM. Detection of C-polysaccharide in Streptococcus pneumoniae in the sputa of pneumonia patients by an enzyme-linked immunosorbent assay. $\ngtr$ Clin Microbiol 1985;22:111-15.

5 Waltman WD, Gray B, McDaniel LS, Briles DE. Crossreactive monoclonal antibodies for diagnosis of pneumoreactive monocional meningitis. $f$ Clin Microbiol 1988;26:1635-40.

6 Pritchard DG, Volonakis JE, Slutsky GM, Greenblatt CL. C-reactive protein binds Leishmania excreted factor. C-reactive protein binds Leishm
Proc Soc Exp Biol 1985;41:500-3.

7 Sugane T, Oshima T. Activation of complement in Creactive protein positive sera by phosphorylcholine-bearing components isolated from parasite extracts. Parasito Immunol 1983;5:385-95.

8 Pery P, Petit A, Poulain JM, Luffan G. Phosphorylcholine bearing components in homogenates of nematodes. Eur $f$ Immunol 1974;4:637-9.

9 Pepys J, Longbottom JL. Antigenic and C-substance activities of related glycopeptides from fungal, parasitic and ities of related glycopeptides from fungal, parasitic
vegetable sources. Int Arch Allergy 1971;41:219-21.

10 Skov Sorensen UB, Henrichsen J. Crossreactions between pneumococci and other streptococci due to C-polysaccharide and F antigen. $\mathcal{F}$ Clin Microbiol 1987;25:1854-9.

11 Mold C, Rodgers CP, Kaplan RL, Gewurtz H. Binding of C-reactive protein to bacteria. Infect Immun 1982;38: 392-5.

12 Sjogren AM, Holmberg H, Krook A. Etiologic diagnosis of pneumonia by antigen detection: crossreactions between pneumococcal C-polysaccharide and oral microorganisms. Diagn Microbiol Infect Dis 1987;6:239-48.

13 Gillespie SH, McWhinney PHM, Patel S, Raynes JG, McAdam KPWJ, Whiley RA, et al. C-polysaccharideMcAdam KPWJ, Whiley RA, et al. C-polysaccharide-
like antigens in alpha-haemolytic streptococci. Infect like antigens in alpha-

14 Parkinson AJ, Rabiego ME, Sepulveda C, Davidson M, Johnson C. Quantitation of pneumococcal C polysaccharide in sputum samples from patients with presumptive pneumococcal pneumonia by enzyme immunoassay. f Clin Microbiol 1992;30:318-22.

15 Krook A, Holmberg H. Pneumococcal antigens in sputa: ELISA for the detection of pneumococcal C-polysaccharide in sputa from pneumonia patients. Diagn Microbio Infect Dis 1987;7:73-5.

16 Ortqvist A, Jonsson I, Kalin M, Krook A. Comparison of three methods for detection of pneumococcal antigen in sputum of patients with community-acquired pneumonia. Eur f Clin Microbiol Infect Dis 1989;8:956-61. 